\title{
Condensin facilitates sister chromatid separation in actively transcribed DNA regions by relieving the obstructive effect of transcription
}

\author{
Norihiko Nakazawa*, Orie Arakawa, and Mitsuhiro Yanagida*
}

Okinawa Institute of Science and Technology Graduate University, G0 Cell Unit, Onna-son, Okinawa, Japan 904-0495.

Short title: Condensin-mediated separation of transcribed DNA

Key words: condensin, chromosome segregation, cell-cycle-regulated transcription, fission yeast, mitosis, heat shock response

*Corresponding authors: nakazawa@oist.jp, myanagid@gmail.com 


\begin{abstract}
The evolutionarily conserved protein complex, condensin, is central to chromosome dynamics, including mitotic chromosome condensation and segregation. Genome-wide localization of condensin is correlated with transcriptional activity; however, the significance of condensin accumulation in transcribed regions remains unclear. Here, we demonstrate that condensin relieves the obstructive effect of mitotic transcription on sister chromatid separation in fission yeast, Schizosaccharomyces pombe. Time-lapse visualization of sister chromatid DNA separation revealed that mutant condensin causes delayed segregation specifically at mitotically transcribed, condensin-bound gene locus, ecm $33^{+}$. Contrarily, the delay was abolished by transcriptional shut-off of the actively transcribed gene. We also showed that delayed separation at a heat shock-inducible gene locus, $s s a 1^{+}$, in condensin mutants was significantly alleviated by deletion of the gene. Since condensin has ability to remove ssDNA-binding proteins and RNA from unwound ssDNAs or DNA-RNA hybrids in vitro, we propose a model that condensin-mediated removal of mitotic transcripts from chromosomal DNA is the primary mechanism of sister chromatid separation.
\end{abstract}




\section{Introduction}

In proliferative cells, mitotic chromosome condensation and subsequent segregation of replicated chromosomes into daughter cells are essential for faithful inheritance of genomic DNA. 'Condensin' has been named as an essential factor for mitotic chromosome condensation, originally demonstrated in mitotic frog egg extracts (Hirano and Mitchison, 1994; Hirano et al., 1997). Today, condensin is known as a key chromosome organizer for many types of chromosome regulation in interphase and mitosis (Hirano, 2016). In fission yeast, Schizosaccharomyces pombe, condensin temperature-sensitive (ts) mutants have been identified by detecting the failure of mitotic chromosome condensation in the same period of condensin discovery in the frog extracts(Hirano and Mitchison, 1994; Saka et al., 1994). Two condensin ts mutants, cut3-477 and cut14-208, were originally isolated, and gene cloning identified two SMC (structural maintenance of chromosomes) family proteins, SMC4/Cut3 and SMC2/Cut14, as the responsible gene products (Hirano, 2006; Saka et al., 1994; Uhlmann, 2016). SMC subunits consist of two globular head domains at the N-and C-termini, which are connected by coiled-coil regions, interrupted by a central hinge region. The condensin Cut3-Cut14 dimer binds to three non-SMC condensin subunits (Cnd2/Barren and HEAT repeat-containing Cnd1 and Cnd3) forming the heteropentameric holo-complex (Sutani et al., 1999; Yoshimura et al., 2002).

Mapping of condensin binding sites at the whole chromosome level proposed a tight correlation between condensin association and transcription. Besides condensin enrichment at centromeres and RNA polymerase I (RNAP I)-transcribed ribosomal DNA (rDNA) repeats (Nakazawa et al., 2008; Wang et al., 2005), genomewide ChIP (Chromatin immunoprecipitation) analyses revealed that condensin associates with RNA polymerase III (RNAP III)-transcribed genes, such as tRNA genes, as well as retrotransposons in budding and fission yeasts (D'Ambrosio et al., 2008; Iwasaki et al., 2010; Tanaka et al., 2012). Fission 
yeast condensin also accumulates at genes highly transcribed by RNA polymerase II (RNAP II) in interphase and mitosis (Nakazawa et al., 2015; Sutani et al., 2015; Toselli-Mollereau et al., 2016). TATA box-binding protein, TBP, and mitotic transcription factors reportedly recruit condensin to RNAP II-transcribed, transcriptionally activated genes (Iwasaki et al., 2015; Kim et al., 2016). Thus, condensin binding at chromosomal DNA accompanies active transcription by RNAPs I, II, and III. Preferential binding to active genes has also been proposed for higher eukaryotic condensin I and II (Dowen et al., 2013; Kim et al., 2013; Kranz et al., 2013; Sutani et al., 2015), and even for prokaryotic condensin (Gruber and Errington, 2009), indicating that transcription-driven condensin accumulation is critical to understand the molecular action of condensin at mitotic chromosomes. However, physiological significance of condensin binding at actively transcribed DNA regions is still enigmatic.

In this study, we performed detailed, time-lapse, live cell analysis of sister chromatid DNA separation during fission yeast mitosis, and demonstrated that condensin ensures sister chromatid separation at mitotically upregulated gene and heat shock-inducible gene loci. Our results provide insight into how mitotic transcription is reconciled with chromosome segregation. 


\section{Results and Discussion}

\section{Sister chromatid DNA separation delays at mitotically transcribed gene locus in condensin mutant}

We have previously shown that $S$. pombe condensin preferentially accumulates at mitotically transcribed genes and heat shock-inducible genes during mitosis (Nakazawa et al., 2015). To understand how condensin contributes to segregate transcriptionally activated regions in mitosis, we examined separation timing of the locus that binds to condensin. To visualise the locus, $7.8 \mathrm{~kb}$ lacO arrays were chromosomally integrated within $2 \mathrm{~kb}$ of the mitotically up-regulated ecm $33^{+}$gene locus (Fig. 1A, left) in wild-type and temperature-sensitive (ts) cut14-208 mutant cells. LacI-GFP was expressed in these cells, and lacO arrays that bound to LacI-GFP were visualized (Nabeshima et al., 1998). lacO arrays were also integrated along the long arm of chromosome 1, within $2 \mathrm{~kb}$ of four other genes $\left(\operatorname{sod} 2^{+}, \mathrm{SPAC} 4 \mathrm{G} 8.03 \mathrm{c}, s c k 1^{+}\right.$, and $\left.n s k 1^{+}\right)$, which were not transcriptionally activated during mitosis. The level of bound condensin was negligible at the four genes (Nakazawa et al., 2015). To visualise the spindle pole body (SPB; equivalent to a centrosome), we used Sad1-mCherry, which is located at the SPBs (Fig. 1A, right) (Hagan and Yanagida, 1995). To monitor the time-course of $\mathrm{lacO}$ segregation, cultures grown at $26^{\circ} \mathrm{C}$ were shifted to $36^{\circ} \mathrm{C}$ (the restrictive temperature for the cut14-208 mutation in the coiled-coil region, S861P) (Sutani and Yanagida, 1997), and $>40$ mitotic cells were analyzed by taking movies for each lacO/LacI-GFP locus in individual wild-type and cut14-208 cells.

Sister GFP loci (green dots) were separated smoothly in wild-type cells, when the SPB distance (between magenta SPB dots) reached 3-4 $\mu \mathrm{m}$ (Fig. 1B, arrows). Sister loci were considered separated when GFP signals were fully split $(>0.5 \mu \mathrm{m})$. SPB distances were then determined at the time point in which the two GFP-dots separated. Measurements from a 
number of movies ( $\sim 50$ cells at each locus, total 635 movies, statistical data is shown in Supplemental Table 1) revealed an inverse relationship between the distance of the loci from the telomere and the mean SPB distance for GFP signal splitting (median bars in Fig. 1C). The telomere-proximal $\operatorname{sod} 2^{+}$locus took longer to split than the centromere-proximal $n s k 1^{+}$ locus.

Representative time-lapse images for cut14-208 condensin mutant cells are shown in Fig. 1D. The SPB distance at the time of sister-locus separation for $\operatorname{sod} 2^{+}$, SPAC4G8.03c, $s c k 1^{+}$, and $n s k 1^{+}$was slightly longer $(4-5 \mu \mathrm{m})$ than in WT $(3-4 \mu \mathrm{m})$. On the other hand, the SPB distance for splitting of the mitotically transcribed ecm $33^{+}$locus was strikingly longer. Splitting did not occur until the SPB distance reached a mean of $6.5 \mu \mathrm{m}$ in mutant cells. Thus, the mitotically highly transcribed $e c m 33^{+}$gene locus showed an anomalous segregation delay in condensin mutant cells, relative to the 4 control loci (Fig. 1E).

\section{Transcriptional shut-off abolishes the delay of sister chromatid DNA separation at actively transcribed genes in condensin mutant}

To examine whether transcription at ecm $33^{+}$affects separation timing at this locus, a promoter-less mutant, $\Delta$ pro ecm 33 , was constructed. ecm $33^{+}$expression was greatly diminished ( $20 \%)$ in $\Delta$ pro ecm33 compared to that in WT cells, as measured by reverse transcription-PCR (Supplemental. Fig. S1). In 4 pro ecm33 cells, the delay of separation at the ecm $33^{+}$locus was essentially abolished (the mean SPB distance became $\sim 5 \mu \mathrm{m}$; Fig. 1E), suggesting an opposing effect of mitotic transcription on sister chromatid DNA separation.

We have also visualized an hsp gene locus, $s s a 1^{+}$, by integrating $l a c O$ arrays $6 \mathrm{~kb}$ upstream of the gene (Fig. 2A). In WT cells, separation timing of the $s s a 1^{+}$-proximal lacO DNAs was not significantly changed in the deletion mutant of $\operatorname{ssal}^{+}(\Delta s s a l)$ after heat shock at $36^{\circ} \mathrm{C}$ (Fig. 2B, Supplemental Table 2). Contrarily, separation timing at that locus became 
significantly earlier by deleting the $s s a I^{+}$gene in cut14-208 (Fig. 2C). We thus concluded that in the condensin mutant, transcription per se caused the delay in sister chromatid DNA splitting at both mitotically-activated and heat-shock responsible gene loci during mitosis.

\section{Condensin-mediated elimination of RNA transcripts may facilitate mitotic chromosome segregation}

The results indicate that condensin negates the inhibitory effect of mitotic transcription on chromosome segregation at actively transcribed gene loci. If we consider in vitro activity of the condensin SMC heterodimer (Cut3-Cut14), it promotes the DNA renaturation reaction which winds up of complementary ssDNA to dsDNA (Sutani and Yanagida, 1997). In addition, a ssDNA-binding protein, Ssb1/RPA, bound to ssDNA, and also RNA molecules hybridized to ssDNA were removed by condensin SMC through the DNA renaturation reaction (Akai et al., 2011). This activity is strikingly reduced in condensin ts mutants, cut14-208 (coiled-coil mutation, S861P) or cut14-yl (hinge mutation, L543S), implying that RNA transcripts bound at actively transcribed regions interfere with chromosome segregation. Transcriptional attenuation using transcription inhibitors or mutation in the transcription mediator protein suppresses chromosome segregation defects in condensin mutants (Supplemental Fig. S2) (Sutani et al., 2015). Taken together, we now propose that condensin-mediated elimination of RNA transcripts on transcribed DNA regions facilitates mitotic chromosome segregation. Actually, many genes are actively transcribed even during mitosis. Our results provide a novel insight into mitotic chromosome segregation, which may be accomplished by removing obstructive RNA and/or proteins on chromosomes, in addition to forming condensed mitotic chromosomes. Further analysis is definitively required to understand the inhibitory effect of bound RNA on chromosome segregation during mitosis. 
Previously, we found that $S$. pombe condensin enriches at central centromeric DNAs where kinetochores are assembled with specialized nucleosomes containing a centromere-specific histone H3 variant, CENP-A/Cnp1 (Allshire and Ekwall, 2015; Nakazawa et al., 2008; Takahashi et al., 2000). In addition to the pericentromeric outer repeat regions (otr), the central centromere also generates a non-coding RNA transcribed by RNAP II (Chan and Wong, 2012; Choi et al., 2011; Talbert and Henikoff, 2018). Condensin enrichment at the central centromere appears to be dependent on transcription (Supplemental Fig. S3), implying that condensin acts on centromeric transcripts. In metaphase, replicated sister centromeric DNAs are pre-separated for establishment of bi-oriented kinetochore-spindle attachment (Nabeshima et al., 1998); however, the pre-separation fails in condensin mutant cells, resulting in unequal segregation of centromere-proximal sister DNAs (Nakazawa et al., 2008). Condensin may play a crucial role in separating centromeres by removing non-coding RNA transcripts, as well as in actively-transcribed chromosomal arm regions. 


\section{Materials and Methods}

\section{Strains, plasmids, and media}

The $S$. pombe haploid wild-type strain $972 h^{-}$and its derivative mutant strains, including the temperature-sensitive (ts) cut14-208, cut3-477 and cnd2-1 were used (Aono et al., 2002; Saka et al., 1994). Disruption of the ecm $33^{+}$promoter (Takada et al., 2010) was carried out using a two-step PCR-based gene targeting method (Bähler et al., 1998). The ecm $33^{+}$gene was cloned into a pSK248 plasmid under the native promoter and expressed in an $e c m 33^{+}$promoter-less mutant to alleviate the cell morphology defect (Takada et al., 2010). Labelling of chromosomal loci at ecm $33^{+}, \operatorname{sod} 2^{+}, \mathrm{SPAC} 4 \mathrm{G} 8.03 \mathrm{c}, s c k 1^{+}, n s k 1^{+}$, and $\mathrm{SSal}^{+}$using the lac repressor $(\mathrm{LacI}) /$ lac operator $(\mathrm{lacO})$ system was performed as previously described (Nabeshima et al., 1998), with the following modifications. The pMK19A-KanR plasmid derived from pMK2A was used for lacO insertion at each locus. pMK19A-KanR contains the $l a c O$ repeat $(7.8 \mathrm{~kb})$ and the kanamycin-resistance gene $\mathrm{Kan}^{R+}(1.7 \mathrm{~kb}$ fragment from pFA6a-KanMX) (Bähler et al., 1998). Target sequences (1.5-2.0 kb) were then cloned into pMK19A-KanR. Resulting plasmids were linearized by cleavage within the target sequences and transformed into a strain expressing the GFP-LacI-NLS protein.

Thiolutin (Sigma T3450) and 1,10-phenanthroline (Sigma 320056) were diluted with dimethyl sulphoxide $(1 \mathrm{mg} / \mathrm{mL})$ and ethanol $(30 \mathrm{mg} / \mathrm{mL})$, respectively, and used to inhibit RNA polymerases I, II, and III (Lackner et al., 2007). Culture media used for S. pombe were YPD and SPA sporulation medium (Saka et al., 1994). Cells were counted using a haematology analyzer (Sysmex FDA-500).

\section{Live cell analysis}

S. pombe cells were cultured at $26^{\circ} \mathrm{C}$ in EMM2 medium and shifted to $36^{\circ} \mathrm{C}$ for 1.5 hr. Before observation, exponentially growing cells were transferred to a glass-bottomed dish 
(IWAKI Glass) coated with $10 \mathrm{mg} / \mathrm{mL}$ concanavalin A (Wako). Time-lapse images were recorded with a DeltaVision microscope system (Applied Precision, GE Healthcare UK Ltd). The objective lens was an oil immersion lens (PlanApo 60x, NA 1.4; Olympus). For observations of lacO/LacI-GFP and Sad1-mCherry signals, a set of images from 7 focal planes at $0.2-\mu \mathrm{m}$ intervals was obtained every $0.5 \mathrm{~min}$. Image projection and deconvolution were performed using an imaging workstation (SoftWoRx; Applied Precision). More than 40 mitotic cells (for Figure. 1) or 25 (for Figure. 2) were observed for each locus. Image J (NIH) was used to measure the SPB distance when lacO/LacI-GFP signals were separated. We defined the separation timing of sister $l a c O /$ LacI-GFP signals when the distance between proximal edges of the GFP signals was greater than $0.5 \mu \mathrm{m}$.

\section{RNA extraction, reverse transcription PCR, and chromatin immunoprecipitation}

Total RNA from $S$. pombe cells was extracted using the hot-phenol method. One $\mu \mathrm{g}$ of total RNA was reverse-transcribed using PrimeScript RT reagent (TaKaRa) with oligo dT primers. A genomic DNA eraser supplied with reverse transcription reagent was used to remove contaminating genomic DNA in the RNA sample. cDNA solution was diluted 25-fold with RNase-free water (Ambion) and $5 \mu \mathrm{L}$ were used for PCR. Results were quantified using real-time PCR with SYBR premix Ex Taq II solution (TaKaRa). Chromatin immunoprecipitation (ChIP) was performed as previously described (Nakazawa et al., 2015). 


\section{References}

Akai, Y., Kurokawa, Y., Nakazawa, N., Tonami-Murakami, Y., Suzuki, Y., Yoshimura, S. H., Iwasaki, H., Shiroiwa, Y., Nakamura, T., Shibata, E., et al. (2011). Opposing role of condensin hinge against replication protein A in mitosis and interphase through promoting DNA annealing. Open Biology 1, 110023.

Allshire, R. C. and Ekwall, K. (2015). Epigenetic Regulation of Chromatin States in Schizosaccharomyces pombe. Cold Spring Harb Perspect Biol 7, a018770.

Aono, N., Sutani, T., Tomonaga, T., Mochida, S. and Yanagida, M. (2002). Cnd2 has dual roles in mitotic condensation and interphase. Nature 417, 197-202.

Bähler, J., Wu, J. Q., Longtine, M. S., Shah, N. G., McKenzie, A., Steever, A. B., Wach, A., Philippsen, P. and Pringle, J. R. (1998). Heterologous modules for efficient and versatile PCR-based gene targeting in Schizosaccharomyces pombe. Yeast 14, 943-951.

Chan, F. L. and Wong, L. H. (2012). Transcription in the maintenance of centromere chromatin identity. Nucleic Acids Res. 40, 11178-11188.

Choi, E. S., Strålfors, A., Castillo, A. G., Durand-Dubief, M., Ekwall, K. and Allshire, R. C. (2011). Identification of noncoding transcripts from within CENP-A chromatin at fission yeast centromeres. J. Biol. Chem. 286, 23600-23607.

D'Ambrosio, C., Schmidt, C. K., Katou, Y., Kelly, G., Itoh, T., Shirahige, K. and Uhlmann, F. (2008). Identification of cis-acting sites for condensin loading onto budding yeast chromosomes. Genes \& Development 22, 2215-2227.

Dowen, J. M., Bilodeau, S., Orlando, D. A., Hübner, M. R., Abraham, B. J., Spector, D. L. and Young, R. A. (2013). Multiple structural maintenance of chromosome complexes at transcriptional regulatory elements. Stem Cell Reports 1, 371-378.

Gruber, S. and Errington, J. (2009). Recruitment of condensin to replication origin regions by ParB/SpoOJ promotes chromosome segregation in B. subtilis. Cell 137, 685-696.

Hagan, I. and Yanagida, M. (1995). The product of the spindle formation gene sad1+ associates with the fission yeast spindle pole body and is essential for viability. J Cell Biol 129, 1033-1047.

Hirano, T. (2006). At the heart of the chromosome: SMC proteins in action. Nat Rev Mol Cell Biol 7, 311-322.

Hirano, T. (2016). Condensin-Based Chromosome Organization from Bacteria to Vertebrates. Cell 164, 847-857.

Hirano, T. and Mitchison, T. J. (1994). A heterodimeric coiled-coil protein required for mitotic chromosome condensation in vitro. Cell 79, 449-458.

Hirano, T., Kobayashi, R. and Hirano, M. (1997). Condensins, chromosome condensation protein complexes containing XCAP-C, XCAP-E and a Xenopus homolog of the Drosophila Barren protein. Cell 89, 511-521. 
Hiraoka, Y., Toda, T. and Yanagida, M. (1984). The NDA3 gene of fission yeast encodes beta-tubulin: a cold-sensitive nda3 mutation reversibly blocks spindle formation and chromosome movement in mitosis. Cell 39, 349-358.

Iwasaki, O., Tanaka, A., Tanizawa, H., Grewal, S. I. S. and Noma, K.-I. (2010). Centromeric localization of dispersed Pol III genes in fission yeast. Mol. Biol. Cell 21, 254-265.

Iwasaki, O., Tanizawa, H., Kim, K.-D., Yokoyama, Y., Corcoran, C. J., Tanaka, A., Skordalakes, E., Showe, L. C. and Noma, K.-I. (2015). Interaction between TBP and Condensin Drives the Organization and Faithful Segregation of Mitotic Chromosomes. Mol. Cell 59, 755-767.

Kim, J. H., Zhang, T., Wong, N. C., Davidson, N., Maksimovic, J., Oshlack, A., Earnshaw, W. C., Kalitsis, P. and Hudson, D. F. (2013). Condensin I associates with structural and gene regulatory regions in vertebrate chromosomes. Nature Communications 4, 2537.

Kim, K.-D., Tanizawa, H., Iwasaki, O. and Noma, K.-I. (2016). Transcription factors mediate condensin recruitment and global chromosomal organization in fission yeast. Nat. Genet. 48, 1242-1252.

Kranz, A.-L., Jiao, C.-Y., Winterkorn, L. H., Albritton, S. E., Kramer, M. and Ercan, S. (2013). Genome-wide analysis of condensin binding in Caenorhabditis elegans. Genome Biol. 14, R112.

Lackner, D. H., Beilharz, T. H., Marguerat, S., Mata, J., Watt, S., Schubert, F., Preiss, T. and Bähler, J. (2007). A network of multiple regulatory layers shapes gene expression in fission yeast. Mol. Cell 26, 145-155.

Nabeshima, K., Nakagawa, T., Straight, A. F., Murray, A., Chikashige, Y., Yamashita, Y. M., Hiraoka, Y. and Yanagida, M. (1998). Dynamics of centromeres during metaphase-anaphase transition in fission yeast: Dis1 is implicated in force balance in metaphase bipolar spindle. Mol. Biol. Cell 9, 3211-3225.

Nakazawa, N., Mehrotra, R., Arakawa, O. and Yanagida, M. (2016). ICRF-193, an anticancer topoisomerase II inhibitor, induces arched telophase spindles that snap, leading to a ploidy increase in fission yeast. Genes Cells 21, 978-993.

Nakazawa, N., Nakamura, T., Kokubu, A., Ebe, M., Nagao, K. and Yanagida, M. (2008). Dissection of the essential steps for condensin accumulation at kinetochores and rDNAs during fission yeast mitosis. J Cell Biol 180, 1115-1131.

Nakazawa, N., Sajiki, K., Xu, X., Villar-Briones, A., Arakawa, O. and Yanagida, M. (2015). RNA pol II transcript abundance controls condensin accumulation at mitotically up-regulated and heat-shock-inducible genes in fission yeast. Genes Cells 20, 481-499.

Saka, Y., Sutani, T., Yamashita, Y., Saitoh, S., Takeuchi, M., Nakaseko, Y. and Yanagida, M. (1994). Fission yeast cut3 and cut14, members of a ubiquitous protein family, are required for chromosome condensation and segregation in mitosis. EMBOJ. 13, 4938-4952. 
Sutani, T. and Yanagida, M. (1997). DNA renaturation activity of the SMC complex implicated in chromosome condensation. Nature 388, 798-801.

Sutani, T., Sakata, T., Nakato, R., Masuda, K., Ishibashi, M., Yamashita, D., Suzuki, Y., Hirano, T., Bando, M. and Shirahige, K. (2015). Condensin targets and reduces unwound DNA structures associated with transcription in mitotic chromosome condensation. Nature Communications 6, 7815.

Sutani, T., Yuasa, T., Tomonaga, T., Dohmae, N., Takio, K. and Yanagida, M. (1999). Fission yeast condensin complex: essential roles of non-SMC subunits for condensation and Cdc2 phosphorylation of Cut3/SMC4. Genes \& Development 13, 2271-2283.

Takada, H., Nishida, A., Domae, M., Kita, A., Yamano, Y., Uchida, A., Ishiwata, S., Fang, Y., Zhou, X., Masuko, T., et al. (2010). The cell surface protein gene ecm33+ is a target of the two transcription factors Atf1 and Mbx1 and negatively regulates Pmk1 MAPK cell integrity signaling in fission yeast. Mol. Biol. Cell 21, 674-685.

Takahashi, K., Chen, E. S. and Yanagida, M. (2000). Requirement of Mis6 centromere connector for localizing a CENP-A-like protein in fission yeast. Science 288, 2215-2219.

Talbert, P. B. and Henikoff, S. (2018). Transcribing Centromeres: Noncoding RNAs and Kinetochore Assembly. Trends Genet. 34, 587-599.

Tanaka, A., Tanizawa, H., Sriswasdi, S., Iwasaki, O., Chatterjee, A. G., Speicher, D. W., Levin, H. L., Noguchi, E. and Noma, K.-I. (2012). Epigenetic regulation of condensin-mediated genome organization during the cell cycle and upon DNA damage through histone H3 lysine 56 acetylation. Mol. Cell 48, 532-546.

Toselli-Mollereau, E., Robellet, X., Fauque, L., Lemaire, S., Schiklenk, C., Klein, C., Hocquet, C., Legros, P., N'Guyen, L., Mouillard, L., et al. (2016). Nucleosome eviction in mitosis assists condensin loading and chromosome condensation. EMBO J. 35, $1565-1581$.

Uhlmann, F. (2016). SMC complexes: from DNA to chromosomes. Nat Rev Mol Cell Biol 17, 399-412.

Wang, B.-D., Eyre, D., Basrai, M., Lichten, M. and Strunnikov, A. (2005). Condensin binding at distinct and specific chromosomal sites in the Saccharomyces cerevisiae genome. Molecular and Cellular Biology 25, 7216-7225.

Yoshimura, S. H., Hizume, K., Murakami, A., Sutani, T., Takeyasu, K. and Yanagida, M. (2002). Condensin architecture and interaction with DNA: regulatory non-SMC subunits bind to the head of SMC heterodimer. Current Biology 12, 508-513. 
bioRxiv preprint doi: https://doi.org/10.1101/644799; this version posted May 24, 2019. The copyright holder for this preprint (which was

not certified by peer review) is the author/funder. All rights reserved. No reuse allowed without permission.

\section{Acknowledgments}

We thank Dr. Steven D. Aird for editing the manuscript. NN was supported by a Grant-in-Aid for Young Scientists (B) 25840013 from the Japan Society for the Promotion of Science (JSPS). We are also grateful for the generous support of OIST. 


\section{Figure Legends}

Figure 1 Delay in sister locus separation at mitotically transcribed $e c m 33^{+}$in condensin mutant, and restoration of the delay by removing the $e c m 33^{+}$promoter

(A) (Left) Schematic diagrams of the ecm $33^{+}$locus and positions of inserted lacO on chromosome 1. Black triangles indicate five distinct positions of the lacO arrays. The distance (in $\mathrm{Mb}$ ) from the left telomere is shown in parentheses under each locus. In the ecm33 promoter-less mutant $\left(4\right.$ pro ecm $\left.33^{+}\right)$, a $0.2 \mathrm{~kb}$ sequence of the 5 '-UTR was replaced with the hygromycin resistance gene. (Right) Scheme for measurement of the distance between two SPBs (bi-directed arrow) that determined the timing of sister locus separation. (B, D) Time-lapse frames of projected images were obtained using a Delta Vision microscope. Loci (lacO/LacI-GFP, green) and SPBs (Sad1-mCherry, magenta) were observed in living wild-type (B) and cut 14-208 (C) mutant cells cultured at $36^{\circ} \mathrm{C}$ for $1.5 \mathrm{~h}$. Selected frames are shown every $1 \mathrm{~min}$. Yellow arrowheads indicate timing of sister GFP locus separation (when signals were separated $>0.5 \mu \mathrm{m})$. Scale bar, $5 \mu \mathrm{m}$. (C, E) Box plots of measured distances between SPBs when sister GFP locus signals were separated. At each locus, movies of more than 40 mitotic cells were analyzed. The center line indicates the median, and the box limits are from the second to the third quartile (25\% to $75 \%$ of the data points). The whiskers extend from the box limits to the 2nd and 98th percentile values, with outliers and far outliers indicated by white circles and squares outside the whiskers, respectively. The $P$ value from a two-tailed Wilcoxon-Mann-Whitney test is shown. Statistical data are provided in Supplemental Table 1. Transcription of $e \mathrm{~cm} 33^{+}$gene caused a delay in sister locus separation during mitosis in the condensin mutant. 
Figure 2 Deletion of a heat shock-inducible $s s a 1^{+}$gene alleviated the delay of sister DNA splitting at the locus in cut14-208 mutant

(A) A diagram of the $\mathrm{ssal}^{+}$locus and the position of the inserted lacO on chromosome 1. For constructing the $\Delta s s a 1$ mutant, the ORF sequence of $s s a 1^{+}$was replaced with the hygromycin resistance gene. (B, C) (Left) Time-lapse frames of projected images were obtained using a Delta Vision microscope. The gene loci (lacO/LacI-GFP, green) and the SPBs (Sad1-mCherry, magenta) were observed in living wild-type (B) and cut14-208 (C) mutant cells cultured at $36^{\circ} \mathrm{C}$ for $1.5 \mathrm{~h}$. Images taken at $1 \mathrm{~min}$ intervals are shown. Yellow arrowheads indicate the timing of sister GFP locus separation (when signals were separated $>0.5 \mu \mathrm{m}$ ). Scale bar, $5 \mu \mathrm{m}$. (Right) Box plots of measured distances between SPBs in wild-type (B) and cut14-208 (C) mutant cells. At each locus, movies of more than 25 mitotic cells were analyzed. The center line indicates the median, and the box limits are from the second to the third quartile $(25 \%$ to $75 \%$ of the data points). The whiskers extend from the box limits to the 2 nd and 98 th percentile values, with outliers and far outliers indicated by white circles and squares outside the whiskers, respectively. $P$ values from a two-tailed Wilcoxon-Mann-Whitney test are shown. Statistical data are provided in Supplemental Table 2. 


\section{Supplemental Figures}

\section{Supplemental Figure S1 The ecm $33^{+}$gene transcript level was reduced in the} promoter-less mutant, $\triangle$ pro ecm $33^{+}$

Transcript levels of ecm $33^{+}$were determined in asynchronously cultured WT and promoter-less mutant $\Delta$ pro ecm $33^{+}$cells using quantitative reverse-transcription (RT)-PCR. Results were obtained using two probes (probes 1 and 2).

\section{Supplemental Figure S2 Partial rescue of temperature-sensitive condensin mutants by transcription inhibitors}

The transcription inhibitors thiolutin and 1,10-phenanthroline partly suppressed the temperature-sensitive (ts) phenotype of cut3-477 and $c n d 2-1$ mutants at $34.5^{\circ} \mathrm{C}$ and $26-33^{\circ} \mathrm{C}$, respectively (arrowheads). Both drugs target RNA polymerases I, II, and III. These data confirm the results of Sutani and colleagues (Sutani et al., 2015).

\section{Supplementary Figure S3 Centromeric association of condensin decreased in the presence of a transcription inhibitor}

ChIP (Chromatin immunoprecipitation)-quantitative PCR analysis of Cut14-FLAG was performed at the centromeric region of chromosome 3 in nda3-KM311 $\beta$-tubulin cs mutants (Hiraoka et al., 1984), along with a non-tagged control strain. (A) Procedure of experiment. Cells were mitotically arrested at $20^{\circ} \mathrm{C}$ for $7 \mathrm{hr}$, and then incubated at $20^{\circ} \mathrm{C}$ for additional $1 \mathrm{hr}$ in the presence of $50 \mu \mathrm{M}$ ICRF-193 (DNA topoisomerase II inhibitor) (Nakazawa et al., 2016), $50 \mu \mathrm{M}$ CPT (Camptothecin, DNA topoisomerase I inhibitor), or $120 \mu \mathrm{g} / \mathrm{ml}$ 1,10-phenanthroline (Transcription inhibitor). DMSO was used for a mock treatment. (B) ChIP results. Positions of PCR primers (arrows with numbers) are indicated in a schematic 
illustration of centromere 3. Relative enrichment was calculated as the ratio of IP to WCE with error bars showing standard deviations $(n=3)$. Association of Cut14 at central centromeric DNA (cnt3 and imr3) was remarkably decreased by transcriptional inhibition, and partly affected by inhibition of DNA topoisomerase II.

\section{Supplemental Table 1}

Statistical data of measured distances between SPBs when sister GFP locus signals separated $\left(\right.$ ecm $33^{+}$, pro ecm $33^{+}$, sod2 $2^{+}$, SPAC4G8.03c, sck1 $1^{+}$, and $n s k 1^{+}$loci).

\section{Supplemental Table 2}

Statistical data of measured distances between SPBs when sister GFP locus signals separated $\left(s s a 1^{+}\right.$and $\Delta s s a 1^{+}$loci). 


\section{B}
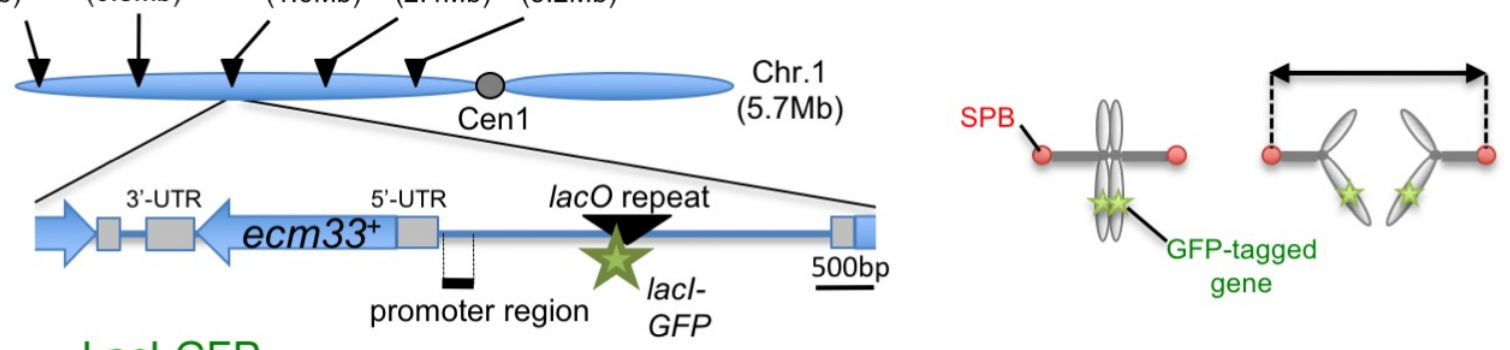

Wild type

Lacl-GFP

Sad1-mCherry $\Delta$ pro.

sod2 $2^{+}$4G8.03c ecm33 $3^{+}$ecm33

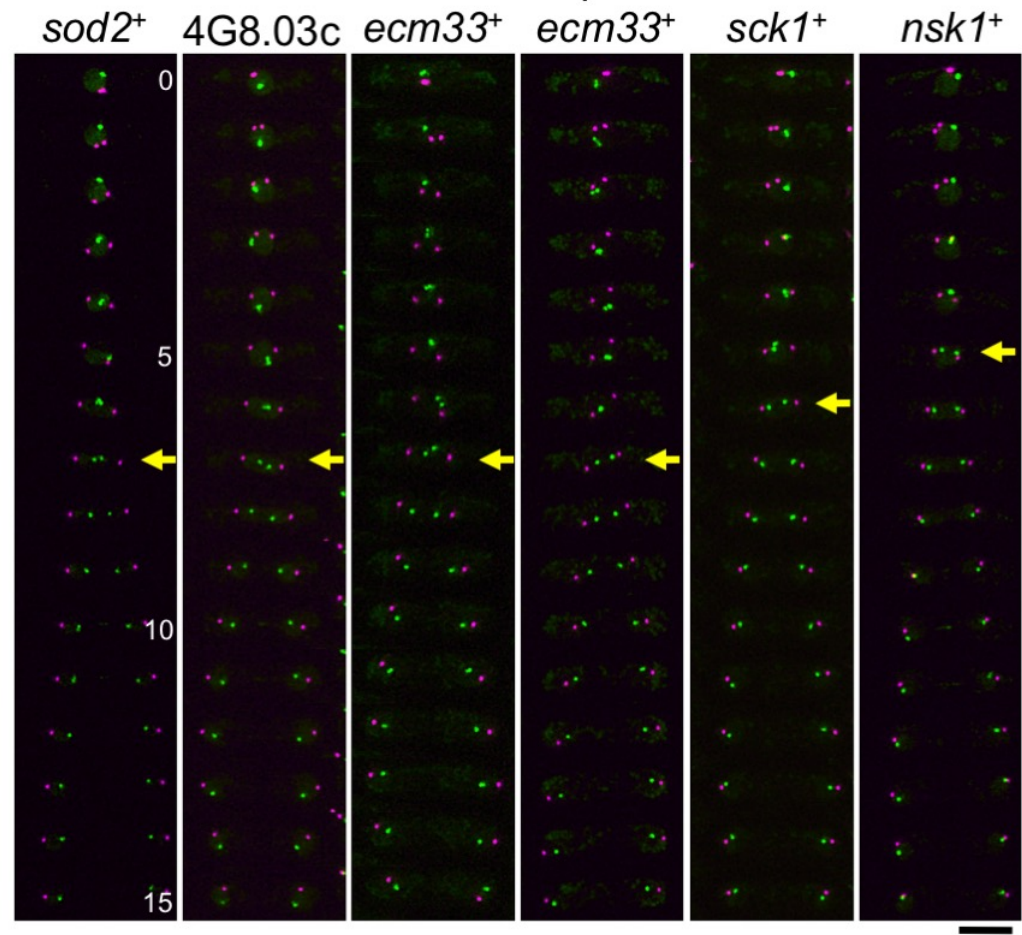

\section{D}

cut14-208

$\operatorname{sod} 2^{+}$

\section{$4 \mathrm{G} 8.03 \mathrm{c} \mathrm{ecm} 33^{+}$}

$\Delta$ pro.

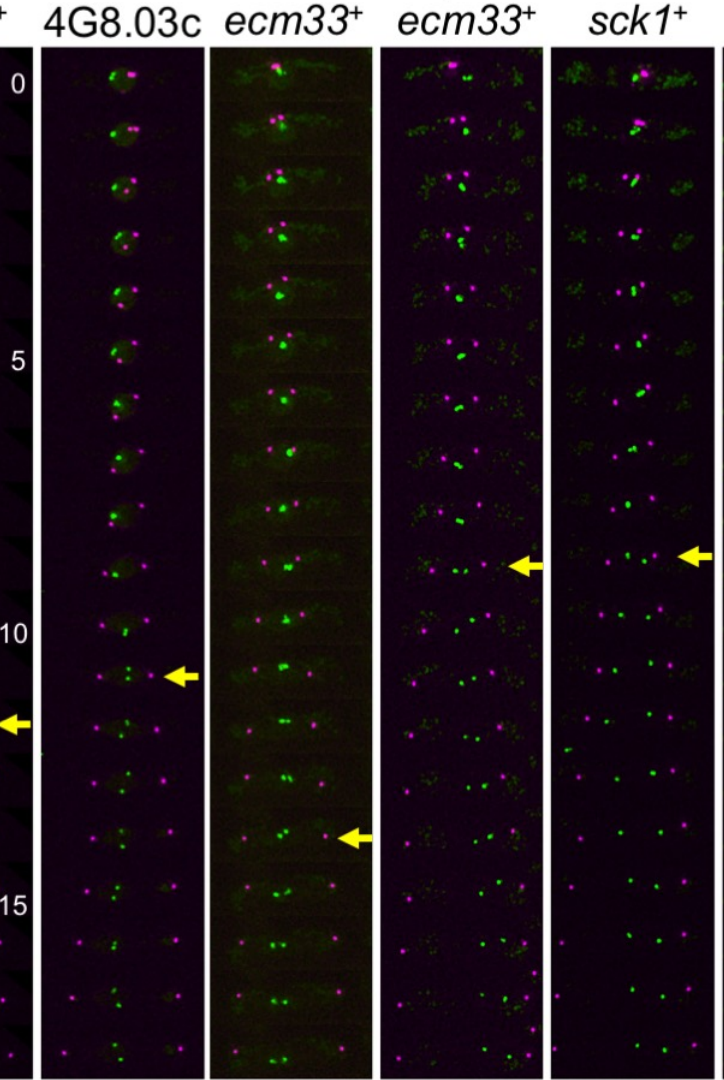

$n s k 1^{+}$

E

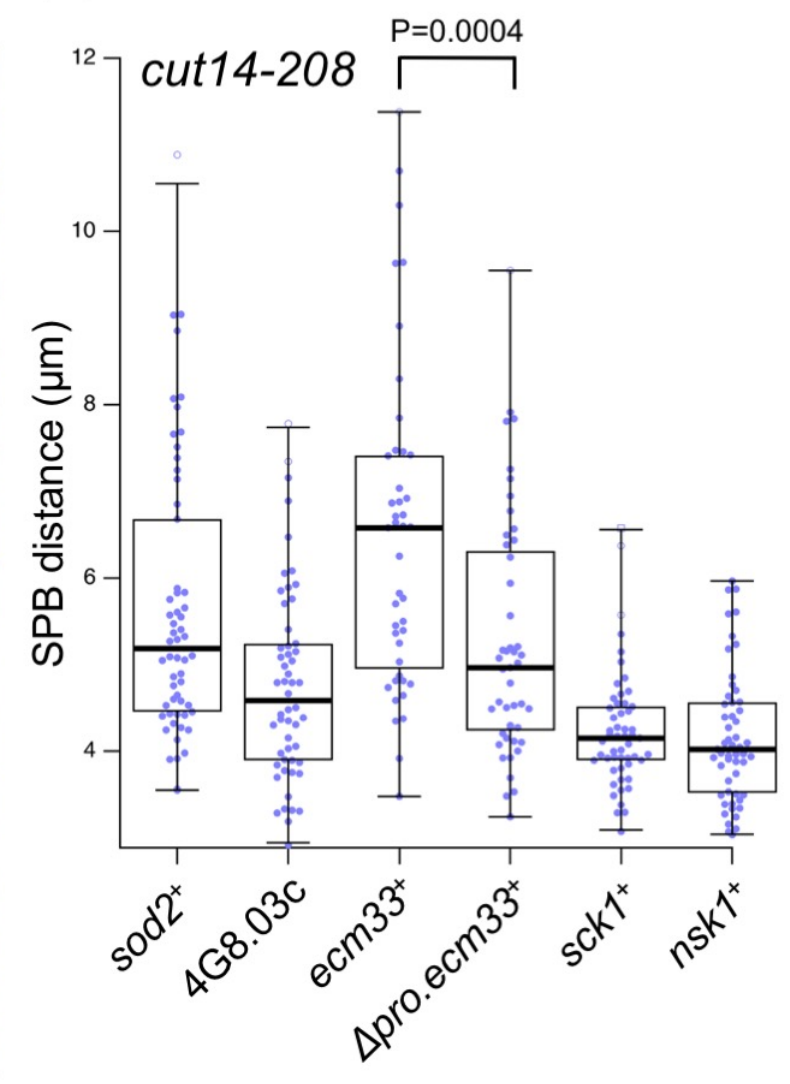

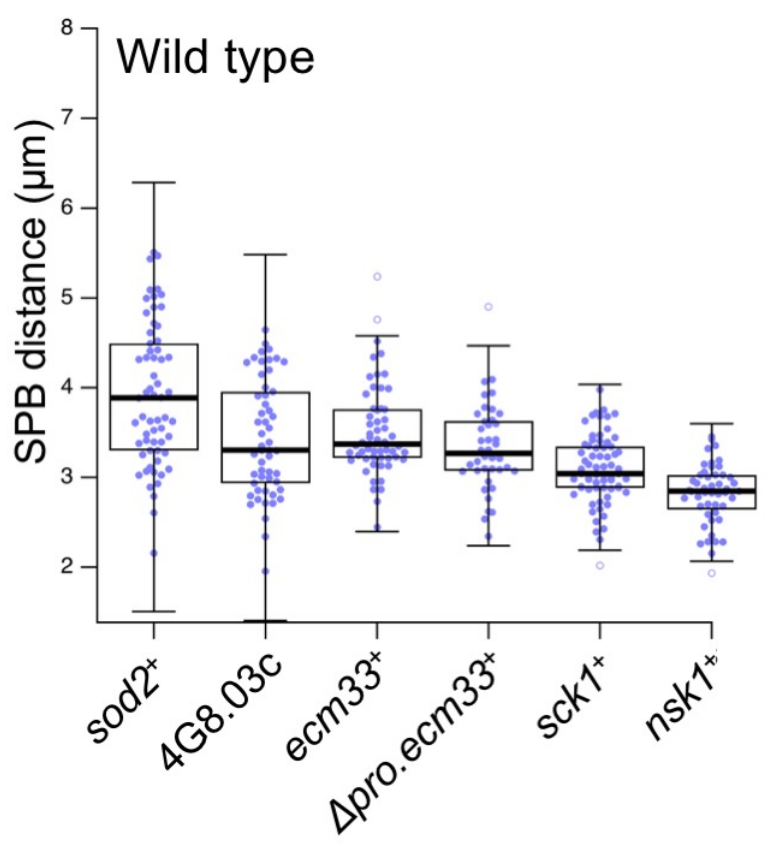

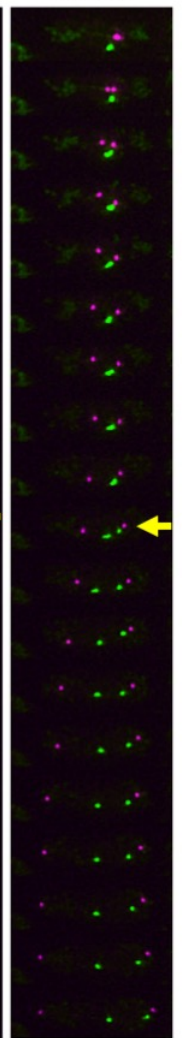


A

LacO repeat at $6 \mathrm{~kb}$ upstream of $s s a 1^{+}$

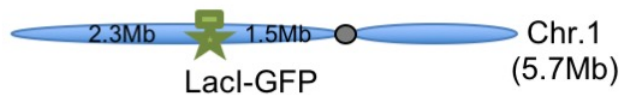

B

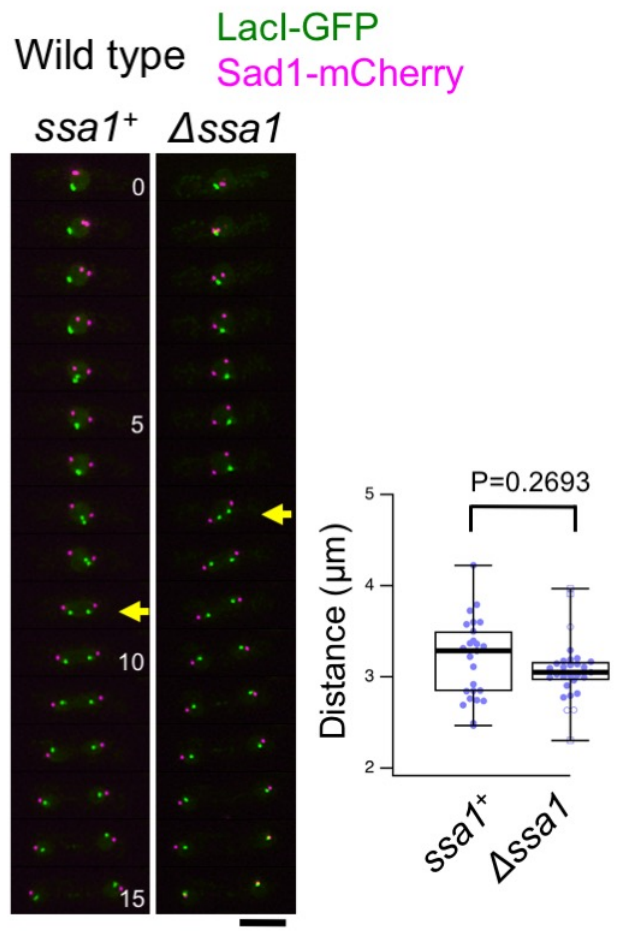

C

cut14-208

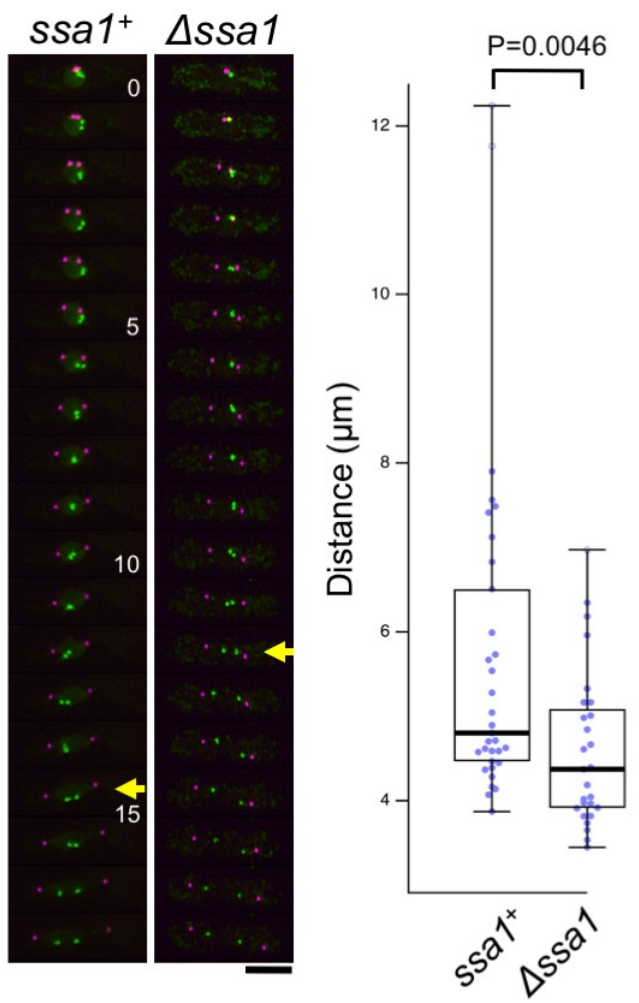




\section{Supplemental Fig. S1}

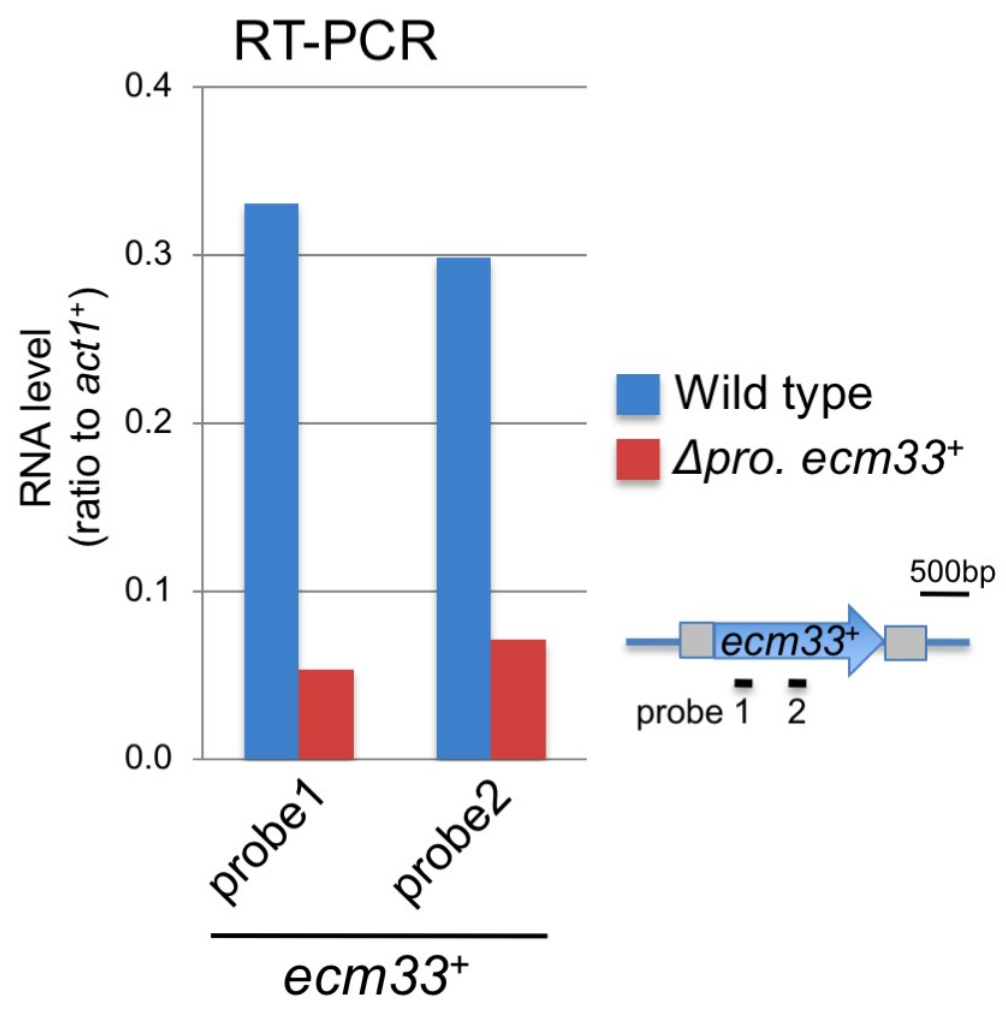




\section{Supplemental Fig. S2}

\begin{tabular}{|c|c|c|c|c|c|}
\hline $\mathbf{A}$ & & Thiolutin & & & \\
\hline & & $0 \mu \mathrm{g} / \mathrm{ml}$ & $0.5 \mu \mathrm{g} / \mathrm{ml}$ & $1 \mu \mathrm{g} / \mathrm{ml}$ & $2 \mu \mathrm{g} / \mathrm{ml}$ \\
\hline & Wild type & 0003 & 0000 得 & 0000 & 0009 \\
\hline & cut3-477 & 0000 能絲 & 000 연 & 0003 & 000 得 \\
\hline & Wild type & 0000 : 4i & $000 \%$ & 0000 & $100 \div$ \\
\hline & cut3-477 & & 00 & 0 & 00 \\
\hline & & 1,10-pher & anthroline & & \\
\hline & & $0 \mu \mathrm{g} / \mathrm{ml}$ & $5 \mu \mathrm{g} / \mathrm{ml}$ & $10 \mu \mathrm{g} / \mathrm{ml}$ & $20 \mu \mathrm{g} / \mathrm{ml}$ \\
\hline $26^{\circ} \mathrm{C}$ & $\begin{array}{c}\text { Wild type } \\
\text { cnd2-1 }\end{array}$ & $\begin{array}{l}0009 \\
00\end{array}$ & 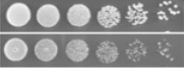 & 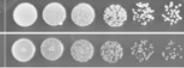 & $\begin{array}{l}00090 \\
0000\end{array}$ \\
\hline $30^{\circ} \mathrm{C}$ & Wild type & 000 이료 & 000 : & 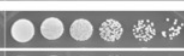 & 000 的的 \\
\hline & cnd2-1 & 0 & 000 & 0008 & 000 \\
\hline${ }^{\circ} \mathrm{C}$ & Wild type & 0000 ㄴ.20 & $0000 \%$ & 00003 & 0000 漛游 \\
\hline & cnd2-1 & & (2) & Ca & 용․ \\
\hline
\end{tabular}




\section{Supplemental Fig. S3}

A $33^{\circ} \mathrm{C}$

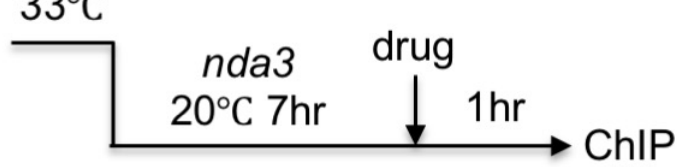

B Centromere 3
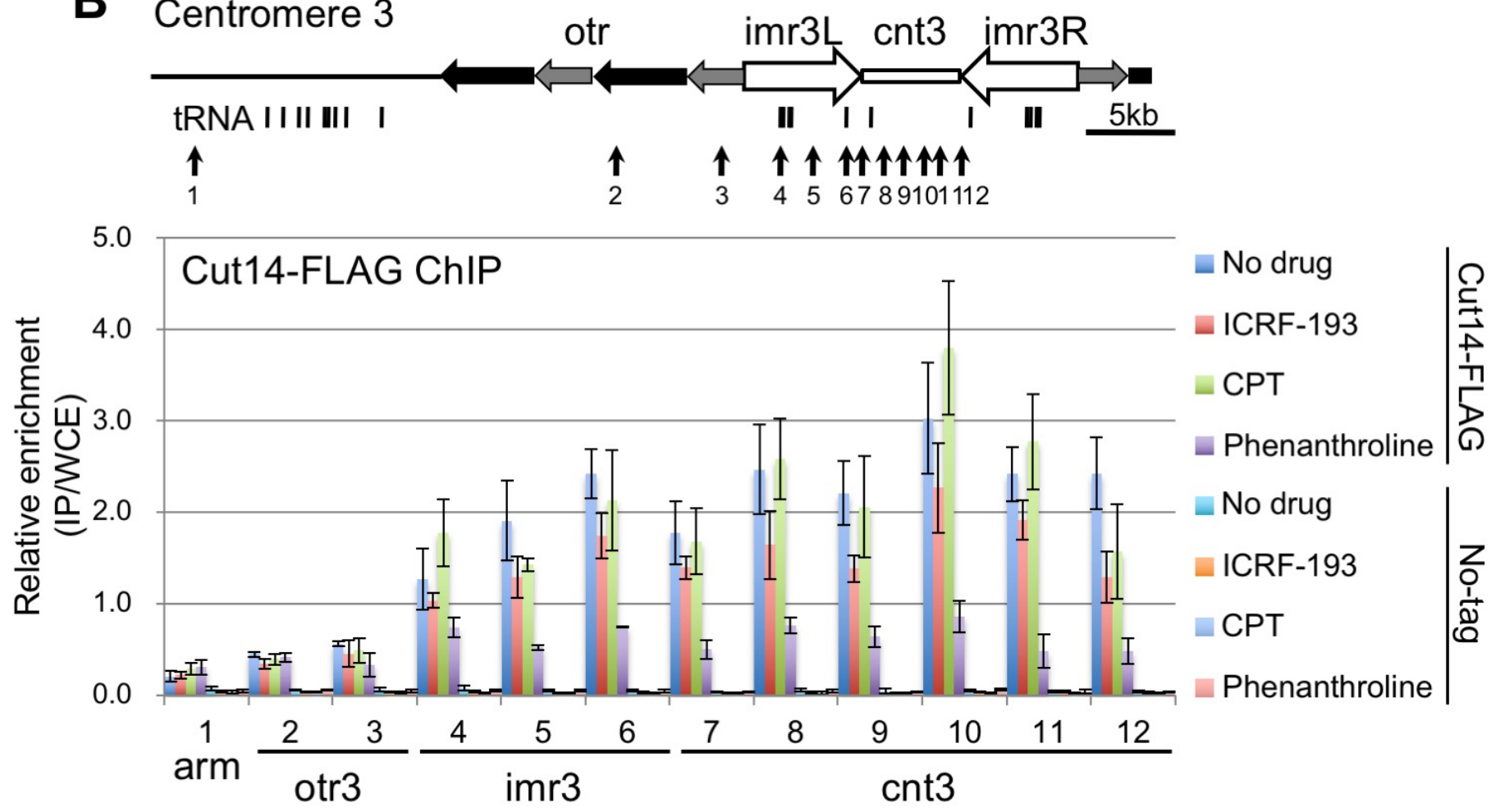


\section{Supplemental Table 1}

Table S1. Statistical data of measured distances between SPBs when sister GFP locus signals were separated $\left(e c m 33^{+}, \Delta\right.$ pro ecm $33^{+}$, sod $2^{+}$, SPAC4G8.03c, sck $1^{+}$, and $n s k 1^{+}$loci)

\begin{tabular}{|c|c|c|c|c|c|c|c|c|c|c|c|c|}
\hline \multirow{2}{*}{$\begin{array}{l}\text { Strain } \\
\text { Locus }\end{array}$} & \multicolumn{6}{|c|}{ Wild type } & \multicolumn{6}{|c|}{ cut14-208 } \\
\hline & $\operatorname{sod} 2^{+}$ & $\begin{array}{c}\text { SPAC } \\
4 \mathrm{G} 8.03 \mathrm{c}\end{array}$ & ecm $33^{+}$ & $\begin{array}{c}\Delta p r o . \\
\text { ecm33 }\end{array}$ & $\operatorname{sck} 1^{+}$ & $n s k 1^{+}$ & $\operatorname{sod} 2^{+}$ & $\begin{array}{c}\text { SPAC } \\
4 \mathrm{G} 8.03 \mathrm{c}\end{array}$ & ecm $33^{+}$ & $\begin{array}{c}\Delta p r o . \\
\text { ecm33 }\end{array}$ & $\operatorname{sck} 1^{+}$ & $n s k 1^{+}$ \\
\hline Number of cells & 61 & 53 & 56 & 40 & 67 & 48 & 58 & 54 & 43 & 47 & 53 & 55 \\
\hline Minimum & 2.16 & 1.96 & 2.44 & 2.34 & 2.02 & 1.93 & 3.55 & 2.91 & 3.48 & 3.24 & 3.07 & 3.03 \\
\hline 25th percentile & 3.30 & 2.94 & 3.22 & 3.07 & 2.88 & 2.66 & 4.48 & 3.90 & 4.95 & 4.24 & 3.89 & 3.52 \\
\hline Median & 3.89 & 3.30 & 3.37 & 3.27 & 3.04 & 2.81 & 5.19 & 4.59 & 6.58 & 4.97 & 4.15 & 4.02 \\
\hline 75th percentile & 4.50 & 3.96 & 3.76 & 3.63 & 3.35 & 3.03 & 6.48 & 5.24 & 7.41 & 6.31 & 4.52 & 4.57 \\
\hline Maximum & 5.51 & 4.65 & 5.24 & 4.90 & 3.98 & 3.45 & 10.88 & 7.78 & 11.38 & 9.55 & 6.58 & 5.97 \\
\hline Average & 3.92 & 3.44 & 3.52 & 3.33 & 3.09 & 2.81 & 5.65 & 4.75 & 6.49 & 5.26 & 4.24 & 4.18 \\
\hline S.D. & 0.79 & 0.65 & 0.51 & 0.49 & 0.38 & 0.34 & 1.60 & 1.12 & 1.87 & 1.40 & 0.68 & 0.80 \\
\hline
\end{tabular}

Time-lapse images were recorded by the DeltaVision microscope system (Applied Precision, GE Healthcare UK Ltd). Image projection and deconvolution were performed using an imaging workstation (SoftWoRx; Applied Precision). Image $\mathrm{J}$ (NIH) was used to measure the SPB distance when lacO/Lacl-GFP signals were separated greater than $0.5 \mu \mathrm{m}$ (Materials and methods). The unit is $\mu \mathrm{m}$ except for number of cells. 


\section{Supplemental Table 2}

Table S2. Statistical data of measured distances between SPBs when sister GFP locus signals were separated (ssa $1^{+}$and $\left.\Delta s s a 1\right)$

\begin{tabular}{lccccc}
\hline \multirow{2}{*}{ Strain } & \multicolumn{2}{c}{ Wild type } & & \multicolumn{2}{c}{ cut14-208 } \\
\cline { 2 - 3 } \cline { 5 - 6 } Locus & $s s a 1^{+}$ & $\Delta s s a 1$ & & $s s a 1^{+}$ & $\Delta s s a 1$ \\
\hline Number of cells & 25 & 29 & & 33 & 27 \\
Minimum & 2.47 & 2.30 & & 3.87 & 3.45 \\
25th percentile & 2.84 & 2.96 & & 4.47 & 3.92 \\
Median & 3.29 & 3.05 & & 4.80 & 4.37 \\
75th percentile & 3.50 & 3.16 & & 6.51 & 5.09 \\
Maximum & 4.22 & 3.97 & & 12.24 & 6.97 \\
Average & 3.19 & 3.07 & & 5.71 & 4.59 \\
S.D. & 0.44 & 0.33 & & 2.00 & 0.93 \\
\hline
\end{tabular}

Time-lapse images were recorded by the DeltaVision microscope system (Applied Precision, GE Healthcare UK Ltd). Image projection and deconvolution were performed using an imaging workstation (SoftWoRx; Applied Precision). Image J (NIH) was used to measure the SPB distance when lacO/Lacl-GFP signals were separated greater than $0.5 \mu \mathrm{m}$ (Materials and methods). The unit is $\mu \mathrm{m}$ except for number of cells. 